
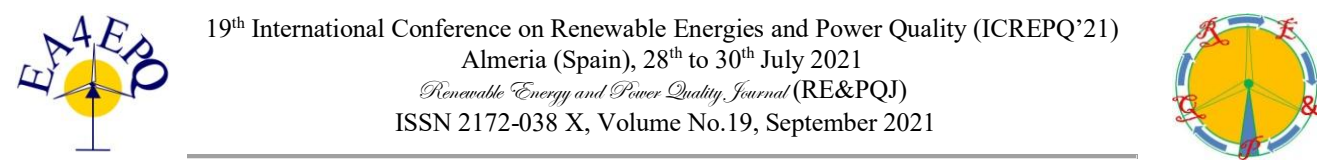

\title{
Fuses in distribution systems: new applications in DC circuits
}

\author{
J. C. Gómez, D. Tourn, C. Reineri, and F. Romero \\ Department of Electrical and Electronic Engineering \\ IPSEP, Rio Cuarto National University \\ Ruta 36, Km. 603, 5800, Río Cuarto, Córdoba (Argentine) \\ Phone/Fax number: +0054 358 4676171, e-mail: jcgomez@ing.unrc.edu.ar
}

\begin{abstract}
The vast majority of distribution systems currently in use, work with alternating current at $50 \mathrm{~Hz}$ or $60 \mathrm{~Hz}$. Several of the distributed resources (generators or storage) supply electrical energy in the form of direct current. Also, there are continuous end-use applications such as centralized variable speed drives, and the rapidly growing application in electric cars which has recently started. This panorama leads to the growing interest in the application of fuses in direct current systems, not as an adaptation of the alternating current fuse but as a specific design. The article presents the crucial differences between direct and alternating circuits, oriented to the operation of the fuse, highlighting their advantages and disadvantages, citing the complications in the design that are required for this growing application. The continuous operation of the fuse is explained in its three fundamental parts: pre-arc, arc and post-arc. The most important current applications are analyzed, such as the protection of: circuits with batteries, circuits of electric vehicles for individual use, power electronics, photovoltaic cells, public transport and circuits in mining. It is concluded in the need to deepen the study of these applications in order to achieve specific fuses designs for direct current and not mere adaptations of the traditional alternating current fuse designs.
\end{abstract}

Key words. Fuses, distribution, direct current, batteries, photovoltaic cells.

\section{Introduction}

The vast majority of distribution systems currently in use operate and have operated since the dawn of electrical distribution with alternating current in $50 \mathrm{~Hz}$ or $60 \mathrm{~Hz}$. Except for a short period, during which the discussion between alternating and continuous, positions defended by Westinghouse and Edison respectively, ends with the imposition of the first, motivated by the invention of the transformer.

At the beginning of the 1990s (more than 35 years ago), an extensive and in-depth investigation was carried out at the IPSEP of the Faculty of Engineering, National University of Río Cuarto on fuses with high breaking capacity to be applied to circuits of low and medium voltage in direct current, for which there was a subsidy from CONICETBID. At that time, the only important field of this type of fuses was for electric traction, whose particular operating characteristics are well marked. After the project was finished, it was thought that the future of this fuse was limited to this application, with a not very promising future due to the slow but constant transformation of these circuits from direct current to alternating current, caused by the advancement of power electronics.

Today, the situation has completely changed, technological advances are reviving research on this type of fuses, mainly due to the trend towards the use of distributed renewable energies (generators or storage) which handle electrical energy in the form of direct current. Also, there are continuous end-use applications such as centralized variable speed drives, and the rapidly growing application in electric cars which has recently started. Part of the electrical energy is beginning to be produced (again) in the vicinity of the point of consumption. This panorama leads to the growing interest in the application of fuses in direct current, not as an adaptation of the alternating current fuse but as a specific development.

The main field of application of the direct current fuse arises from the massive application of three types of equipment: photovoltaic generators (among other generator types), the electric car and large storage batteries. This equipment is directly connected to the distribution system and in some cases is owned or managed by the distribution company. The three equipment types are highly interrelated, since: photovoltaic generation generates continuously and, due to its randomness, requires storage in batteries. On the other hand, the automobile uses pre-stored energy in batteries as an energy source. These new circuits, despite working continuously, like those mentioned for electric traction, have very different characteristics, requiring particular study.

In traditional distribution systems, direct current was used only for auxiliary services, due to its ease of storage in batteries, this being practically the only contact of the electricity distribution professional with direct current. It is apparent that the direct current fuse would be relegated to some circuits such as those corresponding to automobiles, public transport, renewable generation and storage; of no interest to electrical power distribution systems. This concept is totally wrong, since every day there is more correlation between alternating and direct current circuits, both being interconnected by the inverter 
or rectifier, depending on which direction of energy is being studied.

The denomination of direct current in English, DC (direct current) is closer to the current reality than the denomination in Spanish, since in many applications the current is not continuous, but pulsed cyclic unidirectional, fundamentally where semiconductors participate [1].

\section{Characteristics of Direct Current Circuits}

There are crucial differences in the behavior of DC circuits with respect to AC circuits, so the discrepancies oriented to the operation of the fuse will be discussed below, highlighting their advantages and disadvantages, citing the complications in the design that are required for this growing application. In direct current circuits, once the circuit is closed (closing the switch or establishing the fault), the current grows to its final value determined only by the resistance of the circuit, based on an exponential curve whose growth rate is fixed by the time constant of the circuit ( $\tau=\mathrm{L} / \mathrm{R}$, with $\mathrm{L}$ inductance and $\mathrm{R}$ resistance), which is usually measured in ms (milliseconds). This characteristic is a crucial difference with respect to alternating current, which regardless of its final amplitude, will reach its maximum positive or negative value in a time between $5 \mathrm{~ms}$ and $10 \mathrm{~ms}(50 \mathrm{~Hz})$, depending on whether the current is totally symmetrical or asymmetrical (the maximum possible time value for electrical distribution systems is of the order of $8 \mathrm{~ms}$ ). On the other hand, in direct current, a high value of the time constant can cause a slow increase in the current, despite having a high final value. Direct current circuits where fuses are used can have very different time constant values, from extremes of $85 \mathrm{~ms}$ in electric traction (trains, trams and underground) to $1-3 \mathrm{~ms}$ in circuits with photovoltaic cells. Electromagnets, widely used in high power control systems, have exceptionally high time constants, reaching $1,000 \mathrm{~ms}$, cases in which great caution must be exercised with command and protection devices. Between these two extreme values, are the remaining applications in direct current. For clarification purposes, a continuous current with a final unit value, will reach in a time of 5 and $10 \mathrm{~ms}$ (time for the maximum values of alternating current of 50 $\mathrm{Hz}$ ) values of 0.993 or 1 if the time constant is $1 \mathrm{~ms}$, and 0.0055 or 0.0111 if it reaches $85 \mathrm{~ms}$, as can be seen in Figure 1 [2].

This difference in current-time values makes the behavior of the fuses significantly different, elements that act mainly by thermal effect. The analysis presented shows that it is imperative to know the real values of the time constant of the circuit where the fuse will be installed.

On the other hand, the magnitudes of the fault currents in relation to the load current have very different values, with extremes of 20 times in the case of circuits powered by batteries to 1.15 times when the source is a photovoltaic cell, passing for 12 times if powered by a rotary generator. This current relationship is traditionally used as an indication of the presence of a fault in alternating current, seeing that it is not so indicative in the case of direct current applications.

The alternating current passes through zero twice per cycle (in the case of $50 \mathrm{~Hz}$, the first passage through zero may be delayed by the degree of asymmetry of the current, however, in the case of distribution systems it does not take any longer beyond $16 \mathrm{~ms}$ ). These passages through zero are of great importance for the balance "generated heat" to "dissipated heat" and are fundamental in the process of definitive extinction of the current (be it the interruption in a fuse or in a switch of any type). Such passages through zero do not exist for the case of direct current (except in some cases when it comes to current passing through power electronics).

In fuse applications, it is usual to have cyclical loads, which must be taken into account due to their aging effect on the fuse. In direct current applications, the presence of these cyclical charges is much more common than in alternating current, and also their effect is more marked, as will be explained later. Typical cases are for electric traction, with numerous starts, stops, countercurrent and regenerative braking, etc.

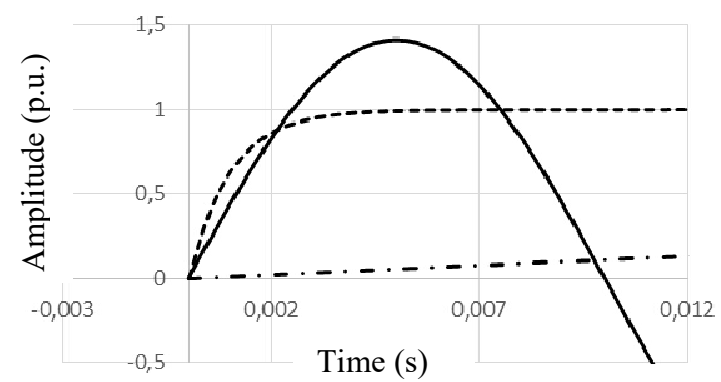

Figure 1, Effect of the time constant on the growth of direct current, compared to alternating current (alternating solid line, continuous dashed $1 \mathrm{~ms}$ and dot-trace $85 \mathrm{~ms}$ ).

\section{Operation of the Fuse in Direct Current Circuits}

The operation of a fuse in a DC system is explained below, in its three fundamental parts: pre-arc, arc and post-arc processes. The principle of operation of the fuse, which is simple in appearance, is based on the melting of a weak link in the circuit when it is traveled by a current greater than normal (pre-arc period). Once the fuse element has melt, an electric arc is established, which must be quenched to achieve the interruption of the abnormal current.

\section{A. Pre-arc process}

The heating and melting processes (if the current is sufficient to reach the melting temperature of the fuse element) are analogous in alternating current and in direct current, being similar when the value of direct current is compared with the effective (rms) value of alternating current. The thermal behavior does not offer differences for the two types of currents. The heating process is based on the interaction between the heat generated (Joule heat based on the equation: $\int \mathrm{r} \mathrm{i}^{2} \mathrm{dt}$ ) and the heat lost by dissipation, in such a way that, if the current is high enough to have a high value of heat generation, the time in which the fusion is reached is sufficiently short as to neglect the losses; A process that is called adiabatic since it is without heat exchange (all the heat generated is used to raise the temperature). The amounts of energy necessary to achieve fusion and complete evaporation are 
constant for each material, depending only on the volume to be melted and evaporated, values known as Mayer's constants. The value of the time after which the fuse stops behaving adiabatically depends on the constructive characteristics of the fuse element, especially on the socalled restriction relationship [3]. The restriction or necking ratio is the quotient between the section of the unrestricted fusible sheet and that corresponding to the restriction, as can be seen from Figure 2, where a typical fusible element is shown.

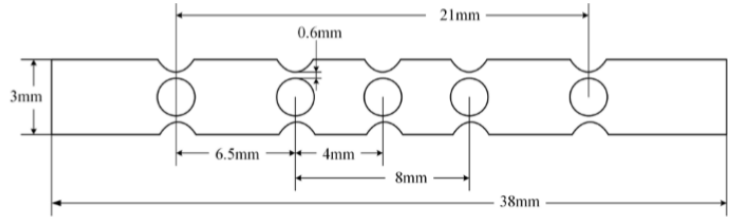

Figure 2, Typical fuse element for direct current and low voltage [4].

The restriction ratio increases as faster operation of the fuse is desired, reaching values of 12 for fuses designed for semiconductor protection, decreasing to 4 for generalpurpose fuses (protection of robust elements such as motors, transformers, conductors, etc.). In direct current applications, there is also the situation of protecting robust and weak equipment, such as batteries and rectifiers or inverters, respectively. The consequence of the wide range of this restriction ratio is that, for fuses with a high restriction ratio, the heating stops being adiabatic for times as short as 1 to $2 \mathrm{~ms}$, a time that extends for less restricted fuses to $100 \mathrm{~ms}$. The time value for which the heating phenomenon ceases to be adiabatic can be determined from the time-current characteristic curve, being the moment at which the curve departs from the straight line

This restriction ratio effect, together with the values of the circuit time constant, lead to a longer pre-arc period duration in DC applications than in the case of alternating current.

It should not be forgotten that, for very high currents, the time constant is irrelevant in the behavior between direct or alternating current.

\section{B. Arc process}

Once the fusion process is over, the pre-arc period has ended and the arc process has been entered, the behavior is totally different from that of the previous period. The quenching of the arc is based on the absorption of energy from the arc in the process of melting the quartz sand that fills the fuse, which transforms into a glass-like structure called fulgurite. The amount of energy per unit volume of this transformation is a constant that depends on the physical-chemical characteristics of the filling material. As the temperature of the filling material is higher, the amount of energy required to form the fulgurite is lower and therefore the ability to extinguish the arc (by extracting its energy) is lower. In other words, it is faster to extinguish the arc when the filling material of the fuse is cold (short pre-arc period) than when it is hot (long pre-arc), being on the other hand, very important the cooling that occurs during zero passages of current in the case of alternating current.
The operation of the fuse (pre-arc and arc) is simpler and safer when the current values are high and grow rapidly, that is, short circuits, which in the case of direct current must also have a low time constant. This simplicity is achieved by the generation of a high arc voltage, which rapidly cancels the current.

The interruption of the low currents presents the aforementioned difficulty, for which a small amount of material with a low melting point is added to the conventional fuse element, shown in Figure 3, which, when exceeding temperatures of 250 to 320 degrees centigrade, dissolves the material of the fusible element, generating a single arc, thus initiating the arcing process. This addition is called M-effect, in honor of its inventor, Metcalf [2].

In this way, it is achieved that the fuses can interrupt currents as low as $60 \%$ above the rated value. This single arc, with the help of the successive passages through zero of the alternating current, is tilting the thermal balance towards the cooling of the arc, concluding with its quenching. If we extend this analysis to the application of the fuse in direct current, the help of the zero passages is not available. The minimum value of current that it is capable of interrupting must rise and is also strongly dependent on the time constant of the circuit.

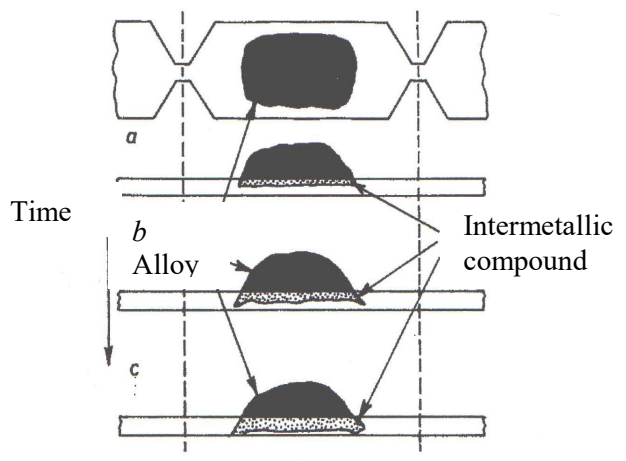

Figure 3, Operation against dissolution overload with M-effect [2].

The solution to the problem of overload interruption with fuses in direct current circuits has been sought by means of constructive aggregates or by means of auxiliary backup devices $[5,6]$. These measures are application dependent and will be discussed below.

\section{Modern Direct Current Applications}

Before starting the topic, it is pertinent to make a warning regarding the use of alternating current fuses in direct current circuits, replacement that must be done strictly following the recommendations of the fuse manufacturer. The manufacturer usually indicates a decrease in the rating value (derating) of the voltage, depending on the time constant of the circuit. There is an empirical rule that indicates that the working voltage in direct current cannot exceed half that of the corresponding one in alternating current, that is, fuses of $500 \mathrm{~V}$ alternating current can be used up to $250 \mathrm{~V}$ direct current [3].

The protection of general equipment with fuses is achieved through the concept of providing through the 
protective device a barrier that interrupts the current before it can damage the protected equipment or shorten its useful life too much. This condition is studied on the time-current and specific energy characteristic curves $\left(\mathrm{I}^{2} \mathrm{t}\right)$ of the fuse and the protected equipment. If the time-current curve of the protector is below and to the left of the protected device's counterpart, any fault current that may occur will cause the protector to operate before causing damage to the protected device. If the fault currents are so high as to consider the phenomenon as adiabatic, the specific energies are directly compared, so that the protector (fuse) has completed the values corresponding to the pre-arc and the total operation, without reaching the amount of $\mathrm{I}^{2} \mathrm{t}$ withstand by protected equipment. Since the time-current and specific energy characteristic curves correspond to elements without use and under normal conditions of application (temperature of $25^{\circ}$ and without preload), it is necessary to take a margin or safety band of the order of 5 to $10 \%$ in terms of current. The presence of cyclical loads may require slightly higher safety factors, in order to avoid the risk of aging, which occurs when the M-effect cumulatively dilutes the fuse element $[2,3]$.

Due to the wide field of application in direct current circuits and differences in particular requirements, these will be dealt with separately in the main applications.

\section{A. Battery circuits}

This application is widely used for UPSs and for the regularization of generation from randomly available sources such as solar and wind. The application is similar to the use in automobiles, but due to other peculiarities of this last use, they will be treated separately.

The breaking capacity of the fuse is crucial in this application, since it is where the highest fault currents are found, reaching values of $100 \mathrm{kA}$, currents that normally have low time constants (of no more than $5 \mathrm{~ms}$ ) facilitating their interruption. These currents, supplied directly by the batteries in cases of failure, without the intervention of power electronics, are called primary currents. Faced with these currents, not only the equipment is at risk but also the risk to personnel and the dreaded occurrence of the explosive arc [7].

Another of the peculiarities of the protection of batteries (or battery banks) is that the energy of the source cannot be considered infinite as is the case of circuits connected to the network, having to act with the least discharge of stored energy. On the other hand, the protection must remain unchanged for the service current, which is obtained as the quotient between the power of the bank and its nominal voltage at the end of the desired discharge period. The operating current and the duration of such current are usually determined for a voltage drop across terminals to $85 \%$. The charging and discharging regime of the bank subjects the fuse to overheating, which, in order to avoid premature aging, requires fuses with a higher rated current, with correction factors that in extreme cases reach $60 \%$. If the fuse is located in a panel or cabinet with reduced dissipation capacity, derating to $70 \%$ may be necessary. When a short-circuit occurs, the protection must act quickly, having as a rule the interruption before two seconds with a fault current equal to 5 times the capacity of the bank in A / h. The analysis presented shows that in many cases the decision will be one of compromise, due to the impossibility of complying with all the requirements. Compliance with these requirements prevents the use of slow fuses (IEC or VDE class gG or gL), and full-field fast fuses must be used, used in semiconductor protection (IEC and VDE class gR) [3].

\section{B. $\quad$ Electric vehicle circuits}

The application of electric cars depends on the capacity of the batteries, which, among other factors, is a function of the voltage of the main traction system, so that the available fault currents are every day greater. To date the voltages of the batteries used in electric cars range from $300 \mathrm{~V}$ to $1,000 \mathrm{~V}$, while traditional vehicles used voltages of $12 \mathrm{~V}$ to $48 \mathrm{~V}$.

In order to avoid a rapid and uncontrolled discharge of stored energy in the event of a vehicle failure or accident, a rapid protective device is required to reduce damage and risk to passengers. The traditional automobile fuse is not capable of fulfilling this function, the obvious solution being to use the industrial fuse, with the necessary adaptations. Safety is seriously affected by the possibility of an electric arc in this primary circuit. There have already been several accidents, in which the vehicle has caught fire and in some cases once the fire is extinguished it is re-ignited due to the availability of remaining energy in the batteries $[6,8]$.

In this application, working conditions very different from those of the industrial fuse are presented, such as ambient temperatures from -40 to $105^{\circ} \mathrm{C}$, which results in correction factors of the nominal current between 1.3 and 0.6. The demanding mechanical conditions such as vibrations and severe shocks should not be forgotten, issues that are not normally considered for the industrial fuse (the exception is the ultra-fast fuses where hardened filler was used due to vibration problems). All of this leads to the need to standardize new tests for standardization.

Cyclic loading is an important factor in studying the behavior of fuses for this application. In the case of industrial devices, overloads were taken into account, for example in the case of motor protection, whose cycles of starting, braking, overloading, countercurrent braking, etc. they were quite predictable and therefore easily considered in device selection. Extremely uncertain overload cycles occur in the automobile circuit, as they are a function of momentary traffic, geographical conditions, weather conditions, driver's driving style, type of vehicle, etc. The battery recharging cycle can also subject the fuse (and consequently the rest of the circuit) to overload regimes, under conditions set by the charge control system. The fuse must be designed for these cyclical overloads without aging occurring or progressing, mainly in the presence of the M-effect or fatigue. This premature aging (shifting of the operating characteristic curves) can cause the fuse to operate under normal load conditions, which may be outside the safe operating zone of the device and also makes it difficult to identify the source of the problem. The solution cannot come with increasing the operating tolerances, since this solution prevents the fuse from performing its protection function.

In general, it can be affirmed that the traditional protection with the conventional fuse is not enough to 
cover the aforementioned requirements, it must be complemented with the aid of external tripping. In other words, the traditional operation of the fuse is maintained in the area of short circuits and severe overloads, where the device has irreplaceable characteristics. For the interruption of slight overloads, extreme overtemperatures, sudden decelerations (car accident), airbag release indication, etc. auxiliary devices are used. For several years, external trip fuse schemes have been presented for industrial applications, these being the first schemes to be applied to electric vehicles. At present, the following schemes can be mentioned: external tripping assisted with fuse with explosive external tripping, shunting the fault current to a parallel path, direct shortcircuiting, etc. Most of the schemes require power from an external source, existing new designs that are selfsufficient, allowing their operation even in the event of complete collapse of the electrical system [5].

The fuse in this application, is no longer a device that responds exclusively to temperature and current-time indirectly, to now have the possibility of external tripping. This addition allows the fuse to have the traditional inverse response curve plus another programmable at will, an idea that is beginning to be used in other applications such as distributed generation circuits, smart grids, etc.

The topic of applying fuses to electric car circuits is still under intense research and design work.

\section{Power electronics}

The devices known as power electronics, have a high load capacity in regime, but are extremely weak to overloads and short circuits (compared to the robustness of the rest of the distribution system equipment), so they require extremely fast protection. To fill this gap, ultra-fast fuses, suitable for their protection, appear simultaneously with electronic devices. Such devices are specified in National and international standards, in the case of Europe by IEC 60269-4 and are called class aR and gR. For technical and economic reasons, it is left to these fuses to protect only against short circuits (greater than 10 times the nominal), the protection against overloads being provided in another way. For this reason, the selection of the fuse is normally based on the comparison of the specific energy values [2]. Depending on the type of power electronics device and the position of the circuit where the fuse is connected, it will be subjected to alternating current, a pulsating current that passes through zero twice per cycle or direct current. The interruption of alternating current is not the subject that is discussed here, the pulsating one passes successively through zero, so the behavior of the fuse is similar to the previous one and in the case of direct current, everything already expressed is valid.

For reasons of protecting such sensitive devices, the current is controlled "electronically", in such a way that frequently the fault currents are of low value, which makes the task of the fuse difficult. It is solved by connecting capacitors (super-capacitors) to the electronics output with values from 2 to $3 \mathrm{~F}$, which provide a discharge pulse that produces the rapid intervention of the fuse, removing it from the risky operating zone [9].

The IEC 60269-4 for semiconductor protection fuses, specifies the breaking capacity and the time constants for the breaking capacity tests, which for fault currents greater than $20 \mathrm{kA}$ should be 10 to $15 \mathrm{~ms}$, instead for lower currents must be equal to $0.5 \times \mathrm{I} \times 0.3 \mathrm{~ms}$ with $\mathrm{I}$ in $\mathrm{A}$.

\section{Solar panel circuits}

Figure 4 shows a simplified diagram of a photovoltaic cell installation.

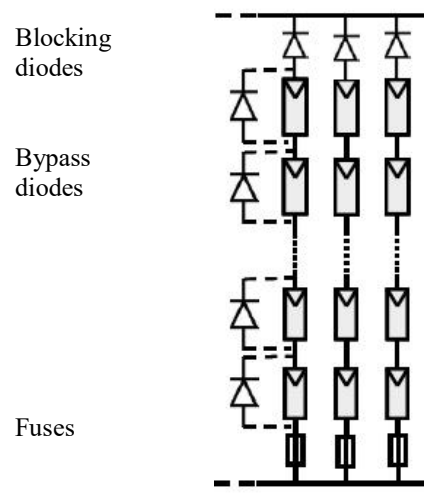

Figure 4, Typical PV group circuit, with three chains with fuses, by-pass diodes and blocking diodes.

It is not necessary to provide protection to the solar panel against direct current (forward), being different for the reverse current that must not exceed the I MOD, REVERSE data given by the manufacturer. The shortcircuit current of the panels is of the order of the direct current under standard conditions, and the current generated under conditions of intense radiation can exceed the corresponding short-circuit STC (Standard Test Conditions) by $40 \%$. Reverse current is due to shade or panel failure, which can cause permanent damage, reduced performance, damage to conductors, and in extreme cases arc and fire. The solution against reverse current is achieved by using fuses installed in series in each chain (necessary as long as there are more than two panels in parallel in each group), adding fuses in each group of panels to avoid damage to the conductors (being recommended a fuse for each conductor, + and -, when the photovoltaic system is isolated from ground).

It should be remembered that the electricity generation of solar panels is highly affected by environmental conditions, so their characteristics are specified for STC, and must be adapted to the actual working conditions under the worst conditions.

For this application, the gPV class fuses standardized by the IEC 60269-6 standard have been developed, specially designed to provide protection against reverse currents, which is not achieved with the other classes of fuses standardized by the IEC 60269-1.

The verification of the breaking capacity of the fuses must always be done, being especially critical in the event that the fuses must interrupt currents coming from large solar farms, batteries or the electrical network.

Due to the difficulty of detecting the operation of fuses, in large installations it is advisable to use blown indicators for each fuse.

For a more detailed description of this protection, there are numerous application guides supplied by the manufacturers of this class of fuses [10].

\section{E. Mass public transport}


The application of fuses in the electrical systems of trains, trams, trolleybuses, etc. has a history of more than 100 years, so its main interruption difficulties are well known. The working voltages range from $700 \mathrm{~V}$ to $1,500 \mathrm{~V}$, with time constants of up to $85 \mathrm{~ms}$ in power circuits and up to $1,000 \mathrm{~ms}$ in the case of actuator coils. There are numerous fuse schemes adapted to each application, using the concept of filling with substances that generate extinguishing gases [1, 2]. These schemes are moving from direct current to alternating current, which, added to the well-known nature of their problems, reduces the importance of their treatment.

\section{F. Mining}

In mining applications, it works with voltages of 300 and $600 \mathrm{~V}$, with fault currents of the order of $35 \mathrm{kA}$ and time constants of up to $30 \mathrm{~ms}$. The main difficulty and the greatest number of problems are found in the interruption of overload currents (200 to $300 \%$ ) due to the length of the galleries and therefore of the supply conductors, with high time constants. This difficulty has led to the appearance of particular designs for this application, in which means to facilitate extinction are incorporated, the most common being the use of substances that generate extinguishing gases, arranged inside the fuse in quite varied ways, according to the manufacturer [11].

\section{Conclusion}

It is concluded in the need to deepen the study of these applications in order to achieve specific fuses designs for direct current and not mere adaptations of the traditional alternating current. It is necessary to extend the knowledge of the behavior of the fuse against variable and repetitive loads, causing thermal-mechanical fatigue. The DC arcing process must be improved through the development of more accurate models and the performance of extensive experimental validations, aimed at ensuring the extinction of low fault currents. It is necessary to understand the effect of the presence of transients that were not normal in alternating current uses, generated by new applications, such as the utilization of fuses in electric vehicles. In addition, there are many new applications with a wide variety of requirements, which generates great efforts to solve these needs, which are very limited in application.

\section{References}

[1] Howe, A., Newbery, G., Nurse, N., DC Fusing in Semiconductor Circuits, IEEE Transactions on Industry Applications, Vol. IA-22, No3, 1986.

[2] Gómez, J. C., Fusibles eléctricos: aplicaciones prácticas y su justificación teórica, EDIGAR, Buenos Aires, Argentina, 2012.

[3] Bessei, H., Manual de Fusibles, $\mathrm{NH} / \mathrm{HH}$, Recycling e.V., Lappersdorf, Alemania, 2012.

[4] Liu, Y., Huang, X., Liu, Z., Peng, J., Xiang, B., Wang J., Gen, Y., Measurement of Current Zero Region Parameters in Current Interruption of DC Current-limiting Fuses, International Conference on Electric Fuses and their Applications, ICEFA, Egaleo, Greece, pp. 1-5, 2019.

[5] vom Dorp, J., Berberich, S., Bauer, A., Ryssel, H., DC-arc behavior of a novel active fuse, ESSDERC 2008 - 38th European Solid-State Device Research Conference, pp. 67-70, 2008.

[6] Koprivšek, M., Triggered Fuse, 10th International Conference on Electrical Fuses and their Applications, ICEFA 2015, Dresden, Germany, September 14th-16th, pp. 69-79, 2015.

[7] Mollet, R., Overcurrent protection of DC power plant equipment using modern high performance current limiting fuses, Proceedings of INTELEC 95. 17th International Telecommunications Energy Conference, 1995.

[8] Rice, C., Nurse, N., Cheong, S., A Study of Challenges for Fuse Link Protection in the New Generations of Environmentally Friendly Vehicles, 9th International Conference on electrical fuses and their application, ICEFA, Maribor, Slvenia, pp. 2-5, 2011.

[9] Giancaterino, J., DC-DC converter plants and their ability to clear distribution fuses, Proceedings of Intelec 94, 11-5, pp. 315-320, 1994.

[10] Photovoltaic System Protection Application Guide, Cooper Bussmann Ltd, United Kingdom, 2012.

[11] Cataltepe, T., Stanek, E., Wiitanen, D., Yenchek, M., Performance and Test Criteria for DC Fuses in Coal Mine Applications, IEEE Transactions on Industry Applications, Vol. IA-22, № 2, 1986. 